\title{
Metastatic transitional cell carcinoma of the bladder in an ileal conduit stoma. A case report and literature review
}

\section{Metástasis de carcinoma de células transicionales de vejiga a estoma del conducto ileal. Reporte de caso y revisión de la literatura}

DCayo Augusto Estigarribia-Benítez,, (DPablo Oteo-Manjavacas, ${ }^{1}$ (DCristina García-Juarranz, ${ }^{1}$ Dinés Hernández, ${ }^{1}$ iDLuis Ignacio Fiter-Gómez. ${ }^{1}$

Keywords:

Bladder cancer,

Metastasis, ileal conduit stoma.
Corresponding author:

*Cayo Augusto Estigarribia Benítez. Carretera Enric Roca Peralta 10 Esc E, $1^{\circ} 1$. CP: 25003 Lleida, España. Correo electrónico: Cayo_med@hotmail.com

\section{Abstract}

Clinical case presentation: An 80-year-old man was admitted to our emergency department due to fever and a progressively growing mass in the urinary diversion stoma, four months after radical cystectomy for muscle-invasive bladder cancer.

Surgical resection was done, and histopathologic examination revealed high-grade urothelial carcinoma metastasis. After nearly three years of follow-up, no signs of recurrence have been seen.

Relevance: Urothelial cancer at all stages can metastasize to other organs.

Hematogenous metastasis spreads to all organs, most frequently to the lungs, liver, or bone and lymphatic metastasis spreads to regional lymph nodes in the pelvis, or further, to the retroperitoneal lymph nodes. Metastatic bladder cancer in the ileal conduit stoma is unusual. Cancer recurrence after radical cystectomy has been reported in ureteroileal anastomoses.

Clinical implications: Surgical treatment is an option in those patients. Adjuvant radiotherapy and/or chemotherapy are possible alternatives in cases of more than one metastasis site. There are no clinical guidelines establishing correct management.

Conclusion: Urinary diversion stoma metastasis secondary to urothelial carcinoma is a rare entity. Direct implantation during the surgical act, or hematogenous, lymphatic, or mixed dissemination, could justify metastatic implantation.

Citation: Estigarribia-Benítez C.A., Oteo-Manjavacas P., García-Juarranz C, Hernández I., Fiter-Gómez L. I. Metastatic transitional cell carcinoma of the bladder to ileal conduit stoma. A Case report and literature review. Rev Mex Urol. 2021;81(2):p: 1-6

${ }^{1}$ Hospital Universitario Severo Ochoa. Leganés, Madrid, España.

Received: May 09, 2020

Aceptado: March 18, 2021

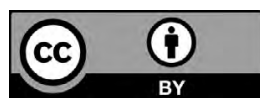




\section{Background}

Bladder cancer heterogeneity is based on its different progression patterns and therapeutic response. Non-papillary tumors have the highest risk for progression to muscle-invasive bladder cancer and metastatic disease. Fifty percent of muscle-invasive bladder cancer (MIBC) develop recurrence after radical cystectomy and distant metastases are more frequent than local recurrence. ${ }^{(1)}$ The most common sites for bladder cancer metastases are the lymph nodes, bones, lungs, liver, and peritoneum. Tumors with a more advanced $\mathrm{T}$ category and an atypical histologic pattern develop early metastases more frequently. ${ }^{(2)}$ The appearance of recurrence in the stoma of the urinary diversion, after radical cystectomy due to urothelial carcinoma, is an extremely rare entity, with very few cases described in the literature. We present herein a case of metastasis in the urinary diversion stoma secondary to transitional cell carcinoma.

\section{Case Presentation}

An 80-year-old man, with a personal history of type 2 diabetes and an ex-smoker, was diagnosed with muscle-invasive bladder carcinoma, pT2G3, and underwent open radical cystectomy and iliac-obturator lymphadenectomy, with a urinary diversion through ureteroileostomy, using the classic Bricker technique, in May 2017. The histologic pattern was high-grade infiltrating urothelial carcinoma, pT3bN0, with surgical margins, ureters, vas deferens, apex, and prostatic urethra free from neoplastic in- filtration. There was no evidence of metastatic lymph node involvement, but images revealed lymphovascular and perineural invasion and an incidental adenocarcinoma of the prostate, Gleason $6(3+3)$ pT1bN0M0. A previous abdominal computed tomography scan was negative for lymph node infiltration or distant metastatic disease.

Four months after surgery, the patient was admitted to the emergency room, presenting with fever and a $3 \times 3.5 \mathrm{~cm}$, exophytic, inflammatory, granulomatous parastomal lesion that progressed in size during admission (Fig. 1-2).

\section{Figure 1. Superficial stomal lesion.}

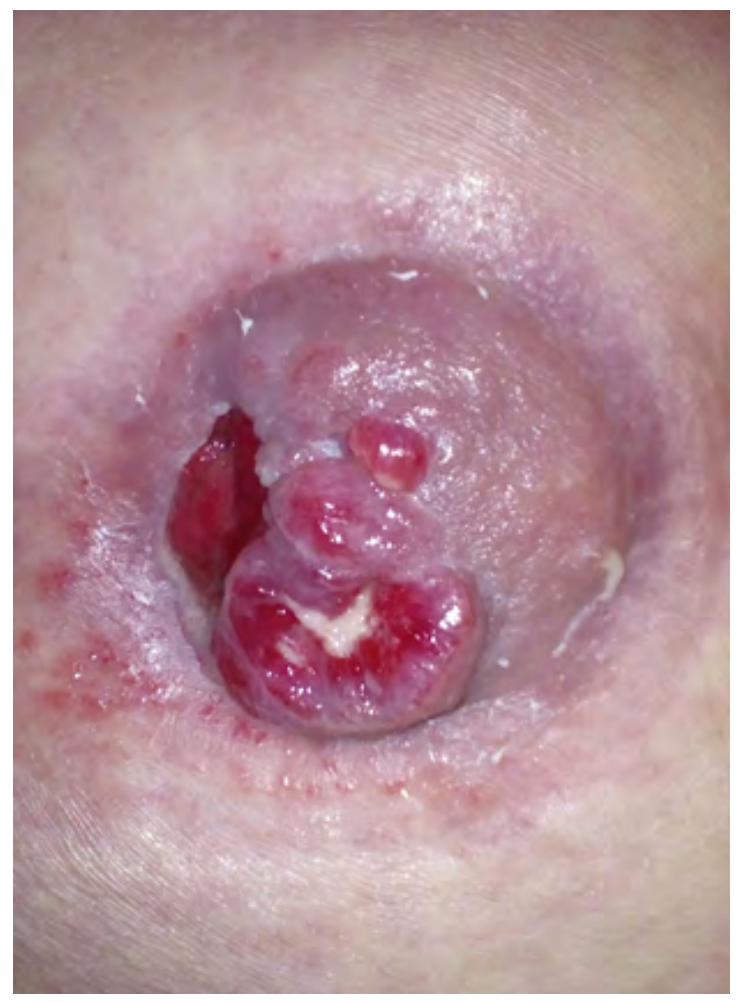

Fig. 1. The lesion appeared ulcerated and bloody, with irregular sites of bleeding. 
Figure 2. Ileal conduit stoma mass.

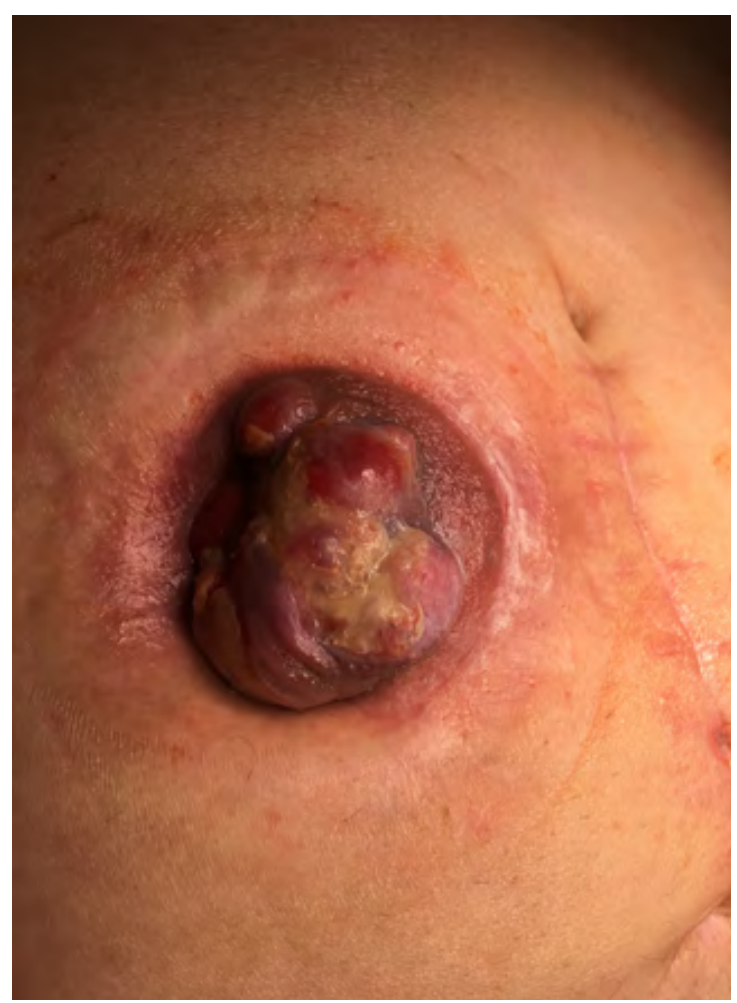

Figure 2. Stoma lesion size increased within a short period of time.

An abdominal computed tomography scan also confirmed a previously undetected subcutaneous $2 \times 3 \mathrm{~cm}$ collection, together with the presence of two pathologic lymph nodes in the right groin. CT urography was performed to evaluate the urinary tract, revealing no apparent lesions.

A biopsy of the lesion was indicated, and results were positive for poorly differentiated urothelial carcinoma. Surgical en bloc resection of the stoma and ileum affected by the tumor was performed, with curative intent. A single lymph node in the mesenteric tissue of the small intestine was also resected (Fig. 3).
Figure 3. Surgical specimen of bladder cancer metastasis.

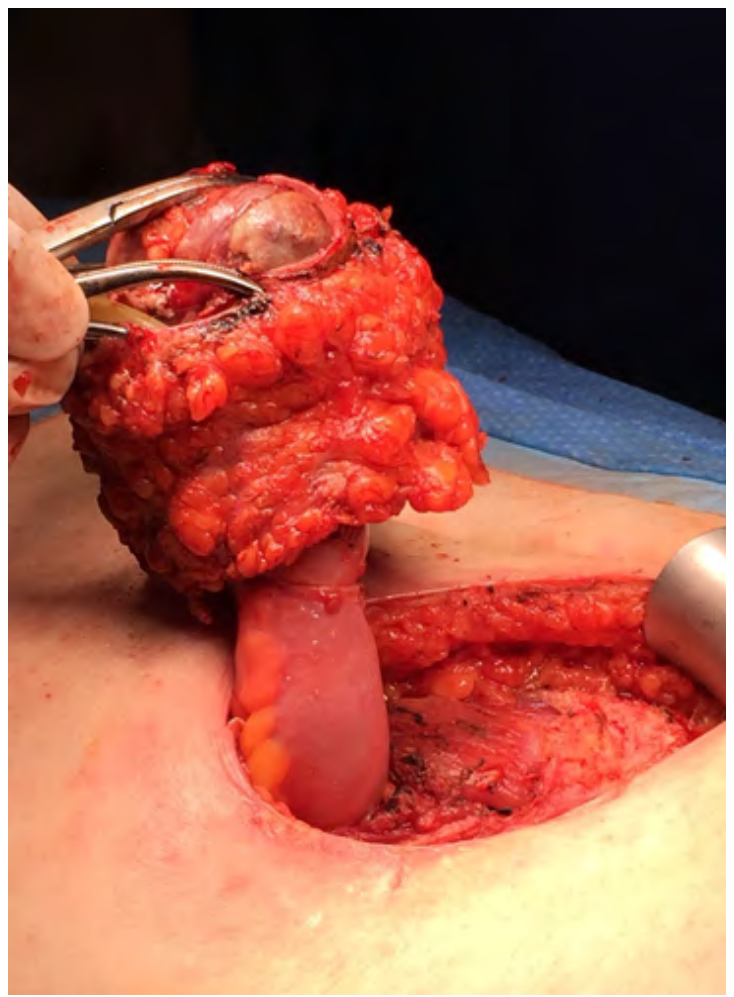

Figure 3. Mass tumoral invasion of subcutaneous tissue and part of the ileal stoma. No portion of the ileum was compromised.

The stoma was reconstructed by advancing the ileum, using the Turnbull technique. The histopathologic exam of the surgical specimen and unique lymph node reported high-grade urothelial carcinoma metastasis, with lymphovascular infiltration, and tumor-free surgical margins (Fig. 4). 
Figure 4. Histologic exam of the surgical specimen

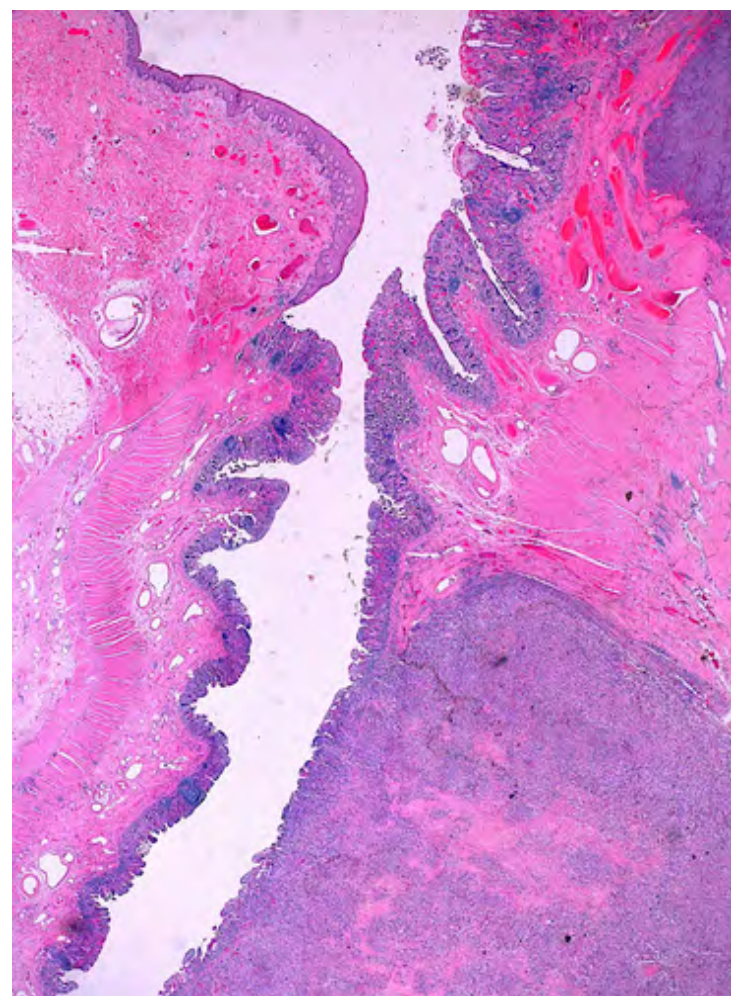

Figure 4. Hematoxylin and eosin-stained longitudinal section of the surgical specimen, with ileal conduit stoma tumor involvement. The left side of the image shows no tumor in that portion of the ileal conduit.

Finally, a biopsy of the groin nodes was performed, two of which were positive for urothelial carcinoma metastasis. The patient did not meet the criteria for chemotherapy because of comorbidity, and so he received treatment with external beam radiation therapy, with excellent tolerance. The patient is currently free of disease, after nearly three years of follow-up.

\section{Discussion}

Recurrence of urothelial tumors in the intestinal diversion after a radical cystectomy is uncommon and is the consequence of local or distant dissemination of the primary tumor. Published cases of recurrence or metastases in ileal conduits after radical cystectomy are rare. ${ }^{(3)}$ In such cases, concomitant recurrence in the upper urinary tract is sometimes found, with ureteroileal anastomosis as the most frequent location. ${ }^{(4)}$ Certain authors argue that this could be due to implantation or seeding during the surgical act, or after the self-implantation of tumor cells from the upper urinary tract. ${ }^{(5)}$ Other studies highlight the multifocal nature of urothelial tumors and their intrinsic tendency to recur. Therefore, the origin of recurrence in the ileal conduit and stoma is likely urothelial tumor recurrence at the level of the distal ureter, growing from the ureteroileal anastomosis and subsequently invading the reservoir. ${ }^{(6)}$ Another study found that a distal ureteral tumor was an independent risk factor for the development of MIBC and radical cystectomy. ${ }^{(7)}$

In 1974, Grabstald described the first case of tumor surrounding the ileal stoma 4 years after a radical cystectomy, assigning the direct implantation of tumor cells during surgery as the probable cause. It was treated with surgical resection, and before performing the urinary diversion, the skin was thoroughly washed and the instruments were replaced. ${ }^{(8)}$ Gupta published a case of squamous cell bladder carcinoma with metastasis to the stoma but with no involvement of the ureteroileal anastomosis, also attributing the implantation of tumor cells during cystectomy as a possible cause. Chemotherapy was the proposed treatment. ${ }^{(9)}$ Takubi presented a case of bladder adenocarcinoma 
Metastatic transitional cell carcinoma of the bladder in an ileal conduit stoma... Estigarribia-Benítez C. A., et al.

recurrence in an ileal stoma 7 months after radical cystectomy, in a patient with previous systemic metastases. Said recurrence was probably due to hematogenous dissemination, as there was no evidence of tumor in the upper urinary tract or in the ileal conduit. ${ }^{(10)}$

To the best of our knowledge, ours is the second case of metastatic urothelial carcinoma in the ileal conduit to be published. The disease emerged early, just 4 months after surgery, possibly due to lymphatic dissemination. There was important lymphovascular compromise (an ileal duct lymph node and right inguinal lymph nodes were positive for malignancy), which was a determining factor for the development of metastasis, as well as an independent predictor of specific and total cancer survival. There was no involvement of the upper urinary tract, ureteroileal anastomosis, or systemic metastasis, and the surgical margins were tumor-free. ${ }^{(11)}$

\section{Conclusion}

Urinary diversion stoma metastasis secondary to urothelial carcinoma is a rare entity. Direct implantation during the surgical act, or hematogenous, lymphatic, or mixed dissemination, could justify the metastatic implantation. Surgical management can be a first approach, with chemotherapy and/or radiotherapy, as possible alternatives in other types of metastases.

\section{Conflict of interest}

None declared.

\section{References}

1. Babjuk M, Böhle A, Burger M, Capoun O, Cohen D, Compérat EM, et al. EAU Guidelines on Non-Muscle-invasive Urothelial Carcinoma of the Bladder: Update 2016. Eur Urol. 2017;71(3):447-61. doi: 10.1016/j. eururo.2016.05.041

2. Shinagare $\mathbf{A B}$, Ramaiya NH, Jagannathan JP, Fennessy FM, Taplin M-E, Van den Abbeele AD. Metastatic pattern of bladder cancer: correlation with the characteristics of the primary tumor. AJR Am J Roentgenol. 2011;196(1):117-22. doi: 10.2214/AJR.10.5036

3. Sánchez Zalabardo D, López Ferrandis J, Arocena García-Tapia J, Sanz Pérez G, Zudaire Bergera J, Berían Polo Jm. Recurrent urothelial tumor in orthotopic neobladder. Actas Urol Esp. 2001;25(8):600-2. doi: 10.1016/s02104806(01)72681-2

4. Barba Abad JF, Tolosa Eizaguirre E, Rincón Mayans A, Berian Polo J. Recidiva tumoral en sustitución vesical ortotópica. A propósito de dos casos y revisión de la literatura médica. Actas Urológicas Españolas. 2010;34(3):295-7. doi: 10.1016/j.acuro.2009.10.002

5. Curran FT, Fuggle WJ. Transitional cell carcinoma in an ileal conduit. Postgraduate Medical Journal. 1986;62(730):769-71. doi: 10.1136/pgmj.62.730.769

6. Rosvanis TK, Rohner TJ, Abt AB. Transitional cell carcinoma in an ileal conduit. Cancer. 1989;63(6):1233-6. doi: https://doi. org / $10.1002 / 1097-0142$ (19890315) 63:6<1233::AID-CNCR2820630633>3.0.CO;2-7

7. Elawdy MM, Osman Y, Taha DE, El-Halwagy S. Muscle-invasive bladder and urethral cancer recurrence after surgical management of upper 


\section{Metastatic transitional cell carcinoma of the bladder in an ileal conduit stoma... Estigarribia-Benítez C. A., et al.}

tract urothelial carcinoma: A review of 305 patients. Turk J Urol. 2018;44(3):213-20. doi: 10.5152/tud.2018.19677

8. Grabstald Harry. Carcinoma of Ileal Bladder Stoma. Journal of Urology. 1974;112(3):332-4. doi: 10.1016/S0022-5347(17)59722-0

9. Gupta C. \& Rajeev K. Ileal Conduit stoma site metastasis in squamous cell carcinoma of urinary bladder. BJUI. 2011 Jun 24.

10. Inobe $\mathbf{T}$, Kanda $\mathbf{K}$, Murakami Y, Tsuji M, Tamura M, Kagawa S. Recurrent bladder adenocarcinoma in an ileal conduit stoma: A case report. International Journal of Urology. 1999;6(9):467-70. doi: https://doi. org/10.1046/j.1442-2042.1999.00094.x

11. Kunju Lakshmi P., You Li, Zhang Yingxi, Daignault Stephanie, Montie James E., Lee Cheryl T. Lymphovascular Invasion of Urothelial Cancer in Matched Transurethral Bladder Tumor Resection and Radical Cystectomy Specimens. Journal of Urology. 2008;180(5):1928-32. doi: 10.1016/j.juro.2008.07.056 